doi:10.17659/01.2017.0044

Journal of Case Reports 2017;7(2):155-157

\title{
Pediatric Presentation of a Celiaco-mesenteric Trunk in Association with a Pelvic Bilateral Renal Ectopia: An Undescribed Association
}

\author{
Edoardo Bindi, Francesco Molinaro, Rossella Angotti, Margherita Aglianò, Maddalena Messina, Mario \\ Messina
}

Department of Medicine, Surgery and Neuroscience, University of Siena, Siena, Italy.

\section{Corresponding Author:}

Dr. Edoardo Bindi

Email: edo.bindi88@hotmail.it

This is an Open Access article distributed under the terms of the Creative Commons Attribution License (creativecommons.org) licenses/by/3.0).

Received : November 22,2016

Accepted : February 6, 2017

Published : April 25, 2017

\begin{abstract}
Background: The celiac trunk presents its normal trifurcation in the $87 \%$ of cases reported in autoptic and radiological studies. Among variants the anatomical conformation known as celiaco-mesenteric trunk has an incidence of $1-2.8 \%$. Case Report: We hereby report a 6 years old girl who presented with recurrent abdominal pain and bilious vomiting. MRI and CT scan, performed with the diagnostic suspicion of an aorto-mesenteric compass, showed the presence of a common origin of the celiac trunk and superior mesenteric artery. In addition, images revealed also the presence of a bilateral pelvic ectopia of kidneys, with renal arteries which took origin from iliac bifurcation. The patient, two months after, was submitted to surgery, during which we found the presence of an intestinal malrotation, instead of an aorto-mesenteric compass. Conclusion: An adequate evaluation of radiologic images and the use of $3 \mathrm{D}$ reconstruction permits to diagnose anatomic variants of celiac-mesentric artery.
\end{abstract}

Keywords: Abdominal Pain, Celiac Artery, Intestinal Volvulus, Mesenteric Artery, Vomiting.

\section{Introduction}

The most important branches of abdominal aorta are the celiac trunk and the superior mesenteric artery, which represent almost the entire vascularization of the visceral territory of gastrointestinal tract [1]. They usually originate respectively from the aorta to the level of T12 and L1. In literature, this conformation is reported only in $87 \%$ of cases, so there are reported many variants [2]. In this work we describe a case of a child affected by Kabuki's syndrome with an intestinal malrotation, carrier of an abnormal conformation of these arterial vessels, in association with a bilateral pelvic ectopia of kidneys.

\section{Case Report}

A 6 year old girl, affected by a Kabuki's syndrome, was admitted to our clinic for abdominal pain and bilious vomiting. Abdominal X-ray and ultrasound were performed and an intestinal occlusion was excluded. A nasogastric tube was placed. An amount of $500 \mathrm{cc}$ of biliary secretion was evacuated in the first 24 hours, with improvement of clinical situation. MRI and CT scan were performed in view of child having repeated similar episodes since birth, with the diagnostic suspicion of an aorto-mesenteric compass. Radiological images were processed with our V-render software and it showed the presence of a common origin of the celiac trunk and superior mesenteric artery. This is known as an anatomic variation called celiacomesenteric trunk. The mesenteric angle was smaller than normal, and it strengthened our suspicion. In addition, images revealed also the presence of a bilateral pelvic ectopia of kidneys. Renal arteries took origin from abdominal aorta at the level of the iliac bifurcation [Fig.1]. Two months later 
we performed a laparotomy, during which it was discovered an intestinal malrotation, instead of an aorto-mesenteric compass. Postoperative course was uneventful, feeding was started on fourth postoperative day, patient was discharged on $7^{\text {th }}$ postoperative day.

\section{Discussion}

The anatomy of the celiac trunk was firstly described by Haller in 1756. However, it is known that the classical conformation is not always present [3]. Many anatomical variants have been reported by Lipshutz in 1917, who firstly described a celiacomesenteric trunk (CMT) [4]. Among variants of celiac trunk, the CMT is the least frequent with an incidence of $1-2.8 \%[6,7]$. In literature, this vascular malformation is reported in association with other anomalies affecting the common hepatic, the left gastric and the splenic arteries [8,9]. Most of papers about CMT, however, are related to series of autoptic and radiological studies in adults [10] because it is usually an asymptomatic anomaly. The association of CMT with a malrotation is to date not described in literature. However this relationship is important because it exposes patient to a risk of a massive volvulus.

The involvement of celiac-mesenteric artery (CMA), indeed, results in necrosis of majority of bowel and of districts of celiac trunk that can have a dramatic impact on a child's life and many long term catastrophic consequences. The aim of this paper is to describe an unique case of CMA associated with abnormal onset of renal arteries that have been well documented by imaging. However, it is important to underline that we made diagnosis of this anatomical variant only for the presence of malrotation.

\section{Conclusion}

An adequate evaluation of radiologic images and the use of $3 \mathrm{D}$ reconstruction permits to diagnose anatomic variant.

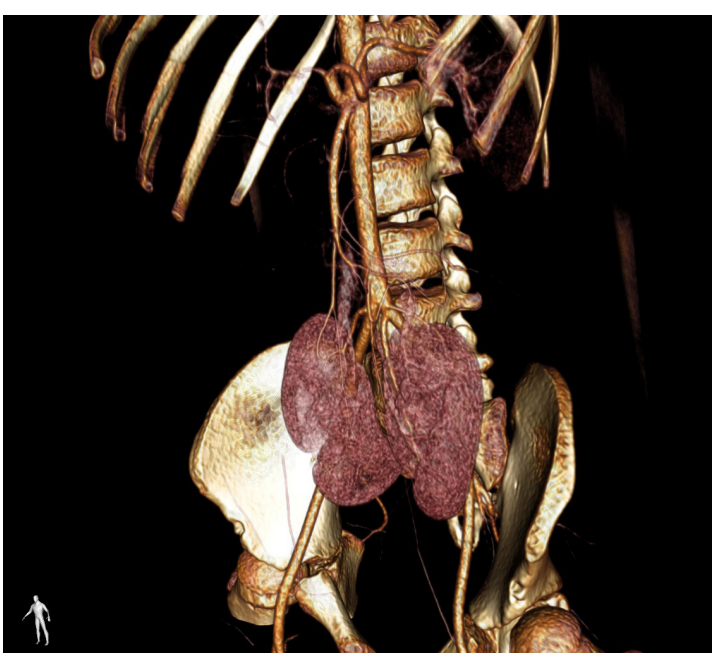

Fig.1: V-Render of TC image: common origin of the celiac trunk and of the superior mesenteric artery, and the pelvic ectopia of kidneys with the renal arteries' origin from the iliac bifurcation.

Contributors: EB, FM: manuscript writing, case management; RA: manuscript editing, case management; MA, MM, MM: critical inputs into the manuscript and literature search. EB will act as guarantor. All authors approved the final version of the manuscript.

Funding: None; Competing interests: None stated.

\section{References}

1. Shuang QY, Hayato T, Munekazu N, Shogo H, Hiroshi M, Akihiko T, Masahiro I. A common celiacomesenteric trunk, and a brief review of the literature. Ann Anat. 2007; 189:482-488.

2. Shorav B, Shorav R, Vishal KJ, Yashwant P, Amar M, Ankur A. Celiaco-mesenteric trunk: a short report. Surg Radiol Anat. 2013;35:979-981.

3. Koops A, Wojciechowski B, Broering DC, Adam G, Krupski-Berdien G. Anatomic variations of the hepatic arteries in 604 selective celiac and superior mesenteric angiographies. Surg Radiol Anat. 2004;26:239-244.

4. Lipshutz B. A composite study of the celiac axis artery. Ann Surg. 1917;65:159-169.

5. Munshi IA, Fusco D, Tashjian D, Kirkwood JR, Polga $\mathrm{J}$, Wait RB. Occlusion of an aberrant right hepatic artery, originating from the superior mesenteric artery, secondary to blunt trauma. J Trauma. 2000;48:325-326.

6. Saeed M, Murshid KR, Rufai AA, Elsayed SE, Sadiq MS. Coexistence of multiple anomalies in the celiacmesenteric arterial system. Clin Anat. 2003;16:30-36.

7. Song SY, Chung JW, Yin YH, Jae HJ, Kim HC, Jeon UB, et al. Celiac axis and common hepatic artery variations 
in 5,002 patients: systematic analysis with spiral CT and DSA. Radiology. 2010;255:278-288.

8. Katagiri H, Ichimura K, Sakai T. A case of celiacomesenteric trunk with some other arterial anomalies in a Japanese woman. Anat Sci Int. 2007;82:53-58.

9. Iezzi R, Cotroneo AR, Giancristofaro D, Santoro M, Storto ML. Multidetector-row CT angiographic imaging of the celiac trunk: anatomy and normal variants. Surg Radiol Anat. 2008;30:303-310.

10. Winston CB, Lee NA, Jarnagin WR, Teitcher J, DeMatteo RP, Fong Y, Blumgart LH. CT angiography for delineationofceliacandsuperiormesentericarteryvariants in patients undergoing hepatobiliary and pancreatic surgery. Am J Roentgenol. 2007;189:W13-W19. 